\title{
Novel Architecture for Wireless Power Transfer in Unmanned Arial Vehicles
}

\author{
Jennifer S. Raj \\ Department of ECE, \\ Gnanamani College of Technology, \\ Namakkal, India \\ E-mail: jennifer.raj@gmail.com
}

\begin{abstract}
There has been a rapid growth in research towards developing smart, low-cost, and high-performance unmanned aerial vehicles (UAVs) for enabling wireless data transfer and communication. The practical applications of UAVs in healthcare, surveillance, agriculture and other domains is increasing at a faster pace. Mass production of these UAVs is essential to meet the growing communication requirements. Plans towards standardization and global deployment of Internetof-Things (IoT) technologies and fifth generation (5G) wireless communication are in progress. Functional diversity is established by the UAVs in enabling wireless communication with IoT and $5 \mathrm{G}$ technologies. The different UAVs used in wireless communication is studied and a hierarchical architecture is proposed to facilitate integrating these technologies into next-generation networks. The principles of aerodynamics, wireless communication, power transfer, area of coverage, delay, meteorological impacts and other design parameters and their tradeoffs is analyzed.
\end{abstract}

Keywords: Unmanned aerial vehicle (UAV), Internet-of-Things (IoT), fifth generation (5G), wireless communication, telecommunication;

\section{Introduction}

Entertainment industry, emergency rescue, security surveillance, agricultural practice, scientific research, industrial inspection, civilian applications, military exploration and various such fields are making use of the benefits of unmanned aerial vehicles (UAVs) leading to a rapid transformation in the domain [1]. Meanwhile, the rapid deployment of Internet of- Things (IoT) and fifth generation (5G) wireless network technologies further enrich the applications and accelerate the diversity and progress of the paradigms. International Telecommunication Union (ITU) speculated application scenarios can be facilitated with the 5G wireless technology aided by UAVs. Massive machine type communications (MMTC), ultra-reliable low latency communications (URLLC), and enhanced mobile broadband (EMBB) are the three principle application scenarios. URLLC is used for handling emergency situations like enhancement of public safety networks, recovery of network service in areas stricken by disaster and so on where UAVs can be deployed to offer efficient outcomes [2]. The network performance can be improved in $5 \mathrm{G}$ cellular network up-to thousand times when compared to that of $4 \mathrm{G}$ network using UAV based EMBB.

Cellular services make use of millimeter wave (mmWave) bands while adopting Upper Microwave Flexible Use Service according to the Federal Communication Committee (FCC) since 2016 [3]. Significant challenges in terms of shadowing effects, huge propagation loss in terrestrial environments are faced by mmWave cellular communication at faster speed and larger bandwidth opportunities. Beamforming schemes can be used despite the high power consumption and hardware resources for overcoming the challenges of propagation loss [4]. In non-line-of-sight (NLoS), the shadowing variance is high and mitigation of mmWave is difficult due to the shadowing effects. The limited channel elements and sparse nature of mmWave channels can be overcome by improving the line-of sight (LoS) communication by deploying wireless networks with UAV technology. Reliable and robust 5G global access is enabled with distinct Earth-space communication with the help of UAV based satellite communication in the near future [5]. 


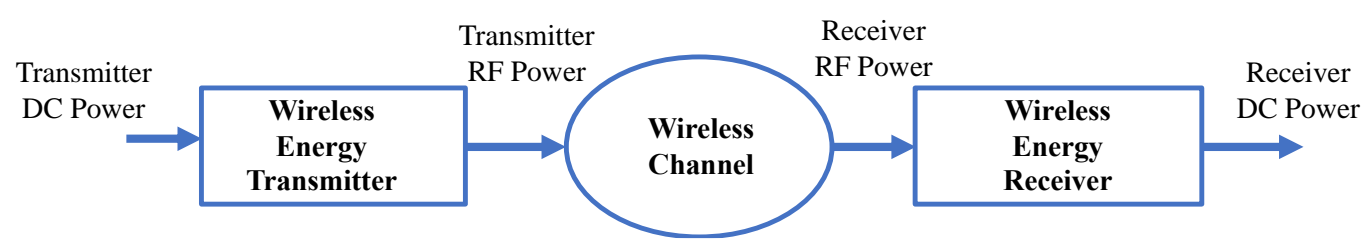

(a)

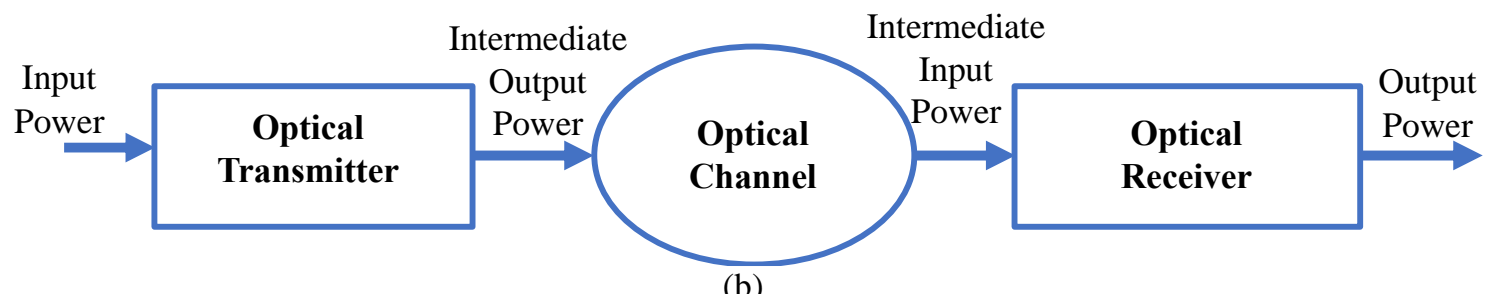

Figure 1: Generic block diagram of (a) Wireless Power Transfer and (b) Laser Power Transfer

Figure 1 (a) represents the block diagram of wireless power transfer through RF system that can operate under the industrial, scientific, and medical (ISM) bands. Figure 1 (b) is the optical wireless power transfer (OWPT) block diagram representing laser power transfer. In this paper, the communication aspects in terms of wireless and aerodynamics under UAV genres is investigated. The weather conditions and corresponding UAV communication channels are analyzed. Further, an optimized and feasible architecture for wireless and laser power transport is proposed.

\section{Literature Review}

Data collection, dissemination, relaying and coverage are the major categories of wireless communication with UAV technology. Relaying of wireless connectivity and offloading can be performed on the 5G base station [6]. Safety concerns caused by UAVs are a major hurdle in the implementation of 5G networks. Several restrictions, rules and laws are implemented by the civil aviation authorities and Federal Aviation Administration (FAA) for operation of commercial UAVs in order to regulate the people, vehicles, construction, distance from airports, speed, altitude and weight. Several technical bottlenecks like on-board power and other significant limitations are also faced by the design of UAVs [7]. A lithium polymer battery of size 1 pound can be used for powering a mini UAV of 3 pounds. For a 30 minute flight time, this battery can provide up to $80 \mathrm{Wh}$ of energy.

The security and latency of the system is outstanding. However, the power requirements of wireless hardware and additional payload necessities are created while installing the UAVs with 5G wireless communication features [8]. This will reduce the duration of operation further. The quality of wireless communication of the and operating time of UAV will also be affected greatly by the adverse weather conditions. The energy consumption for communication of an aircraft engine is higher when compared to that of a micro or pico-cell. The energy efficiency, flight time and robustness of the UAV communication systems are enhanced by several technological solutions. 5G-oriented UAV network implementation can be accelerated by several energy harvesting schemes and novel energy systems [9]. The solar cells operating efficiency is improved by maximum power tracking scheme using UAV and aircraft solar power management technique. Laser power beam schemes are used for improving the storage and energy transfer efficiency with no need for refueling or landing the flight for up to 24 hours. The performance of wireless computation and laser energy harvesting are balanced using a throughput maximization technique [10]. 


\section{Proposed Architecture}

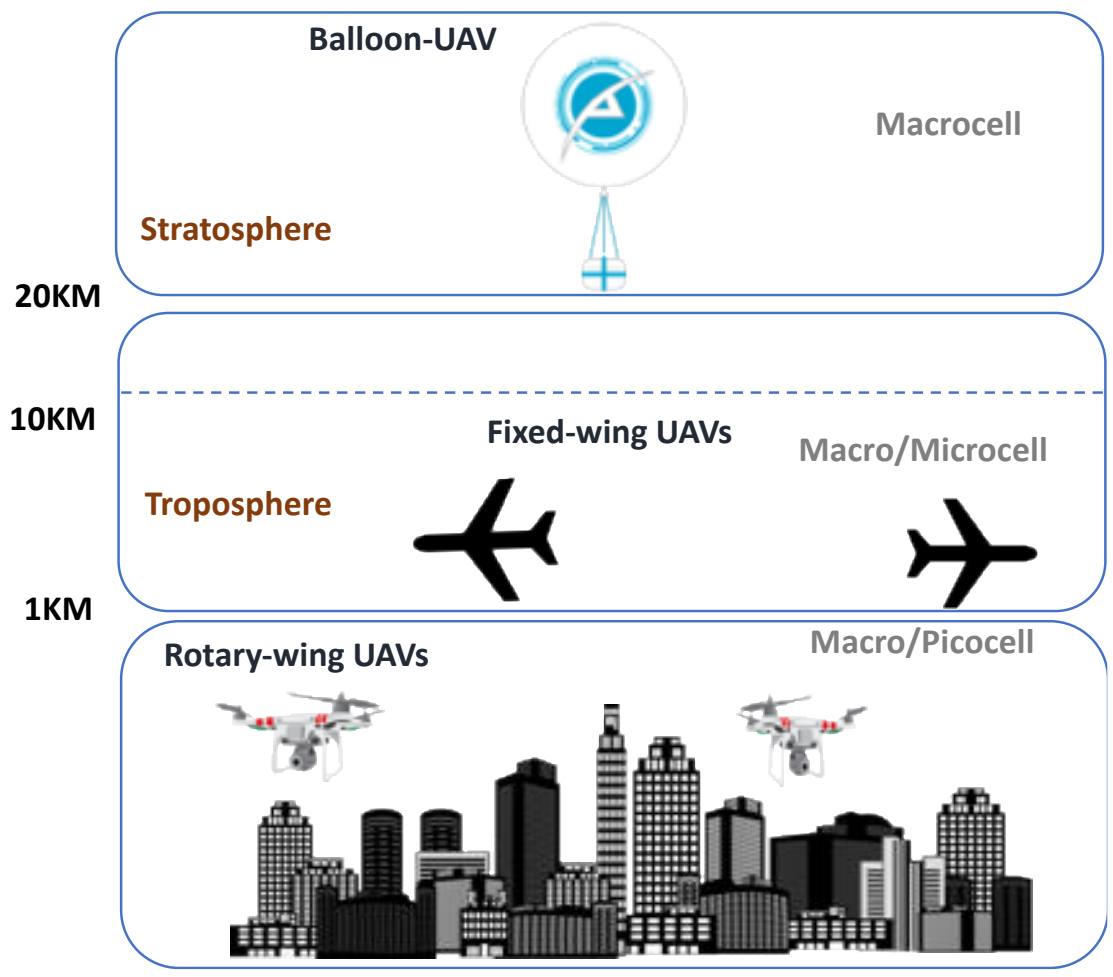

Figure 2: Multilayer distributed UAV architecture

Longest flight duration with heavy load carrying capacity is the major benefit of balloon UAV. This feature is exploited in this paper for long duration hovering while carrying heavy $5 \mathrm{G}$ equipment [11]. The balloon UAV can act as an energy-efficient $5 \mathrm{G}$ macrocell base station covering a radius of $20 \mathrm{Kms}$ weighing hundreds of Kgs. Line of sight links of higher probability can be enabled by high altitude. Figure 2 represents a multilayer distributed UAV architecture based on the various UAV characteristics. The UAV can fly under flexible altitudes with lightweight $5 \mathrm{G}$ equipment.

The UAV balloon is located $20 \mathrm{Kms}$ above the sea level at the stratosphere and behave like quasi-stationary cellular towers [12]. Recycling and relaunch of the balloons can be performed after proper maintenance over a longer time duration. Fixed wing UAVs are positioned above $1 \mathrm{Km}$ and below 10Kms. This helps in addressing the design challenges and minimizing the doppler shift. These UAVs can cruise at very slow speed. The rotary wing UAVs serve as base stations with small cells termed as micro or pico-cells. Quick replenishing and recharging of these UAVs can be performed at low cruise altitudes. The power transfer, energy harvesting and 5G communication is analyzed among different layers. The balloon UAVs are integrated with control and nonpayload communication systems and 5G macro-cell communications [13]. Backwards compatibility and 5G new radio features are offered by the balloons while enabling communication with the ground and areal base stations. Fixed wing and rotary wing UAVs behave as micro/macro or pico-cells.

Far field and near field wireless power transfer schemes are used for various applications. These techniques have different features and functionalities. [14] Laser charging technology, solar chagring, wireless and wired charging, increase of battery capacity and other enhancements help in improving that battery life and overcoming the poor endurance of UAVs. Magnetically-coupled resonant wireless power transfer (MCR-WPT) is used for its application flexibility and operation safety in several applications. The sensor information from the UAVs must also be gathered to offer location stability. Various UAV stabilization algorithms are used for this purpose based on the application for minimizing vibrations.

\section{Results and Discussion}

Various parameters are analyzed to ensure the performance of the proposed model in terms of transmission efficiency, maximum power transfer and meteorological conditions. Figure 3 represents the maximum transmission efficiency of 1500 and $800 \mathrm{~nm}$ over a distance of $10 \mathrm{Km}$ with different visibility scenarios. Under clean air, the transmission is better, however, under fog condition, and multiple temperature ranges, laser 
transmission helps in achieving improved power transmission over different distances. Multiple power transfer frequencies are also considered for omnidirectional transmission. The $800 \mathrm{~nm}$ technology is outperformed by the $1500 \mathrm{~nm}$ providing better transmission efficiency especially in lower temperature. When the transmission distance is beyond 500 meters, a minimum of $10 \%$ transmission efficiency is maintained. The efficiency of laser power transfer may be degraded with medium fog over a distance of more than 200 meters and thick fog over a distance of 50 meters.

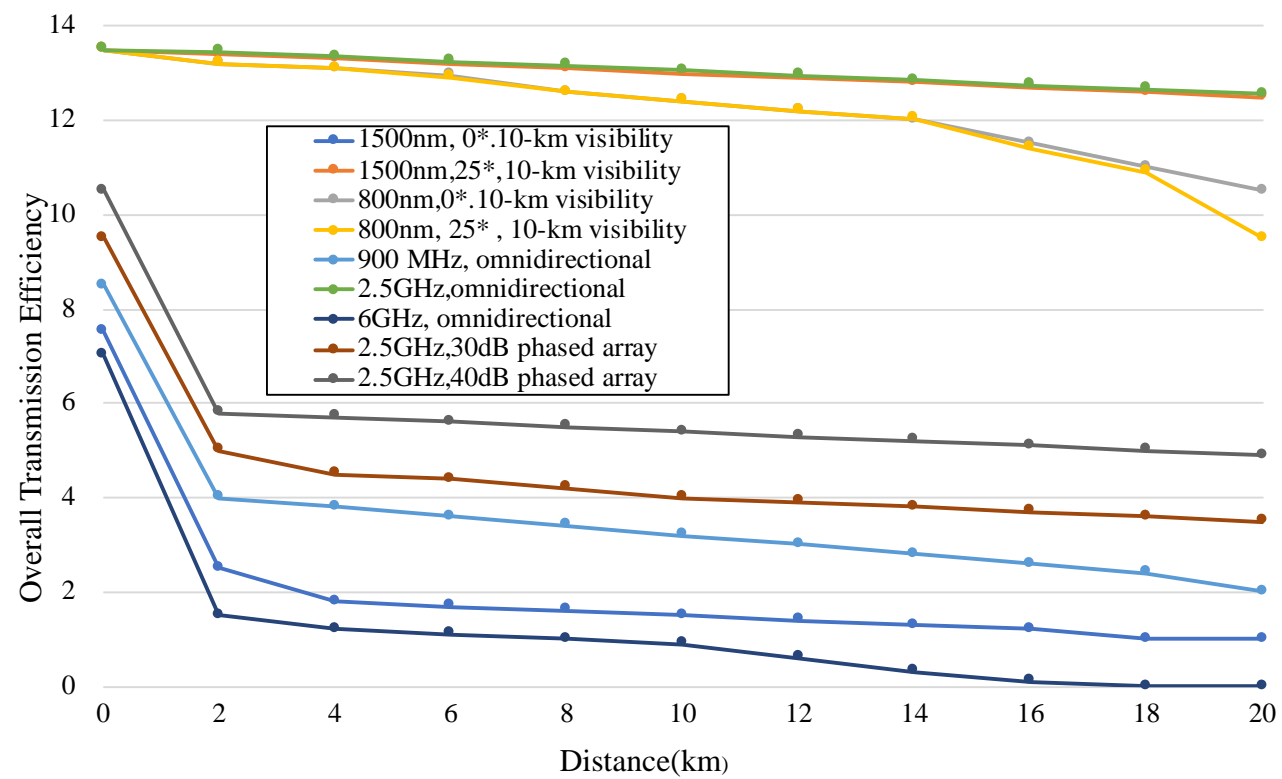

Figure 3: Comparison of transmission efficiency between laser and wireless power transfer

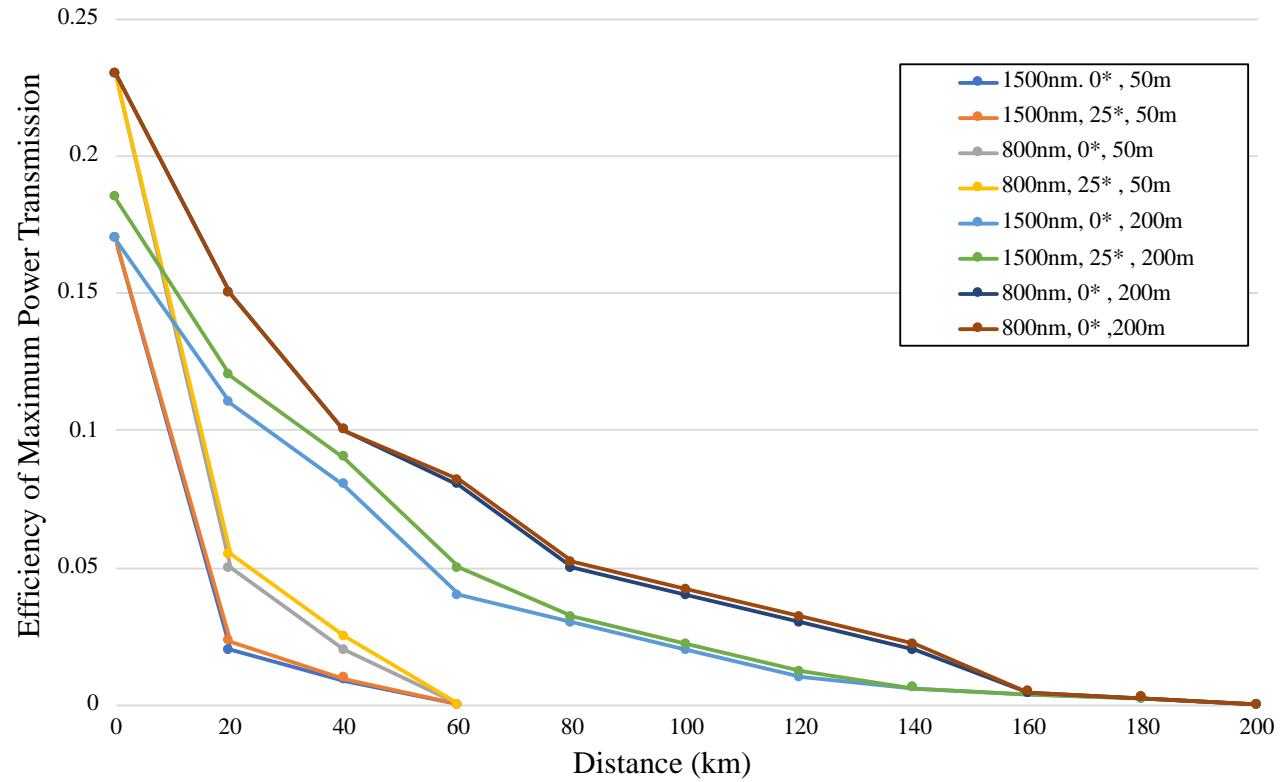

Figure 4: Efficiency of maximum power transmission over multiple distances

Figure 4 represents the efficiency of maximum power transmission over multiple distances namely 50 and 200 meters. In case of rain, heavy snow and abrupt climatic variations, the attenuation of the system varies between 60 to $100 \mathrm{~dB} / \mathrm{km}$. Distance and weather largely affects the power transfer techniques. However, the laser power transfer technique is largely advantageous when compared to the microwave or RF power transfer systems. Different gain factors are considered for the link to enhance the efficiency of transmission. Figure 5 provides the attenuation at various meteorological conditions. The features are analyzed for both phased array and omnidirectional antenna. 
J. Sustain. Wireless Syst., vol. 02, no. 4, pp. 165-170

https://doi.org/10.36548/jsws.2020.4.005

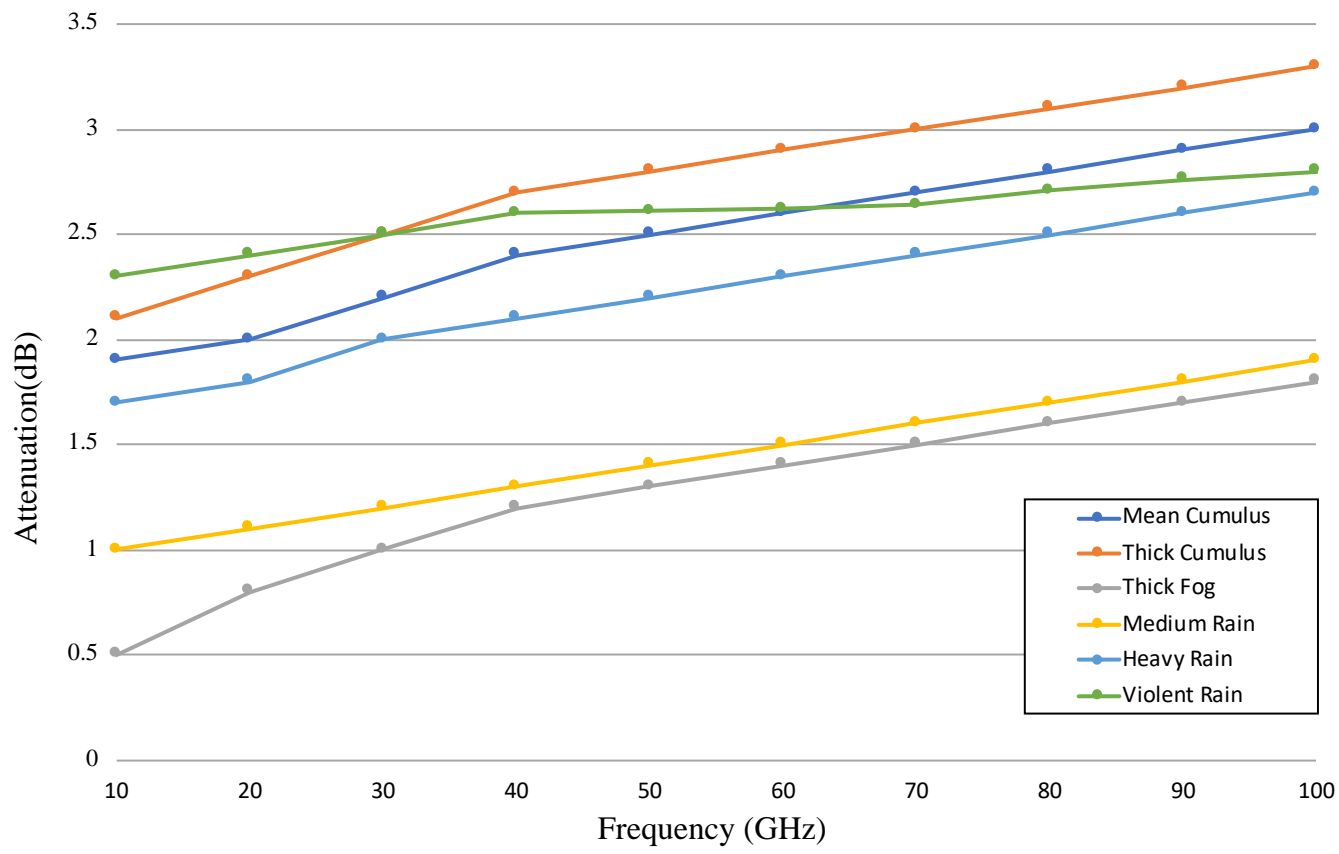

Figure 5: Different meteorological conditions and corresponding attenuation

\section{Conclusion and Future Scope}

Wired power transfer technology may be completely replaced by the WPT technology with the rapid technological advancements. There are promising opportunities for 5G communication with UAV technology. Meteorological variable, aerodynamic constraints, wireless communication, power transfer and other critical factors can be addressed by the integration of $5 \mathrm{G}$ communication with UAV technology. Based on specific application and deployment circumstances, reconfiguration of the flexible and hierarchical networking architecture can be performed. The numerical analysis of the system robustness design and link budget calculation is done with respect to the meteorological conditions, propagation attenuation and laser transmission efficiency. Future work is directed towards hierarchical network management and secure communication design for UAVs, management of communication in adverse weather conditions, integration of deep learning technology and computer vision for secure laser power transfer, precise modeling of non-stationary UAV features and channel propagation, co-design of power transfer, wireless communication sum rate maximization and interference cancellation. Reduction of power loss due to axial or angular misalignment and high oscillation frequency is also to be considered. Further, the physical elements such as resonator, circuits and so on are to be improved at their design level to offer improved and secure communication.

\section{References}

[1] Yang, C., He, Y., Qu, H., Wu, J., Hou, Z., Lin, Z., \& Cai, C. (2019). Analysis, design and implement of asymmetric coupled wireless power transfer systems for unmanned aerial vehicles. AIP Advances, 9(2), 025206.

[2] Vincent, D., Huynh, P. S., Patnaik, L., \& Williamson, S. S. (2018, June). Prospects of capacitive wireless power transfer (c-wpt) for unmanned aerial vehicles. In 2018 IEEE PELS Workshop on Emerging Technologies: Wireless Power Transfer (Wow) (pp. 1-5). IEEE.

[3] Le, A., Truong, L., Quyen, T., Nguyen, C., \& Nguyen, M. (2020). Wireless power transfer near-field technologies for unmanned aerial vehicles (uavs): A review. EAI Endorsed Transactions on Industrial Networks and Intelligent Systems, 7(22).

[4] Junaid, A. B., Konoiko, A., Zweiri, Y., Sahinkaya, M. N., \& Seneviratne, L. (2017). Autonomous wireless self-charging for multi-rotor unmanned aerial vehicles. Energies, 10(6), 803.

[5] Ke, D., Liu, C., Jiang, C., \& Zhao, F. (2017, December). Design of an effective wireless air charging system for electric unmanned aerial vehicles. In IECON 2017-43rd Annual Conference of the IEEE Industrial Electronics Society (pp. 6949-6954). IEEE.

[6] Chen, J., Ghannam, R., Imran, M., \& Heidari, H. (2018, October). Wireless power transfer for 3d printed unmanned aerial vehicle (uav) systems. In 2018 IEEE Asia Pacific Conference on Postgraduate Research in Microelectronics and Electronics (PrimeAsia) (pp. 72-76). IEEE. 
[7] Kumar, S., \& Jayprakash, G. K. M. (2017). Wireless power transfer for unmanned aerial vehicle (UAV) charging. Int. Res. J. Eng. Technol., 4(8), 1939-1942.

[8] Park, J., Kim, J., Shin, Y., Park, B., Kim, W. S., Cheong, S. J., \& Ahn, S. (2019). Toroidal-shaped coils for a wireless power transfer system for an unmanned aerial vehicle. Journal of Electromagnetic Engineering and Science, 19(1), 48-55.

[9] Obayashi, S., Kanekiyo, Y., Nishizawa, K., \& Kusada, H. (2019, June). 85-kHz band 450-W Inductive Power Transfer for Unmanned Aerial Vehicle Wireless Charging Port. In 2019 IEEE Wireless Power Transfer Conference (WPTC) (pp. 80-84). IEEE.

[10] Plaizier, G. M., Andersen, E., Truong, B., He, X., Roundy, S., \& Leang, K. K. (2018, May). Design, modeling, and analysis of inductive resonant coupling wireless power transfer for micro aerial vehicles (MAVs). In 2018 IEEE International Conference on Robotics and Automation (ICRA) (pp. 1-6). IEEE.

[11] Park, B., Park, J., Shin, Y., Park, C., Ahn, S., Han, I. S., ... \& Lee, K. S. (2017). Wireless Charging System Using Soft Magnetic Composite for Unmanned Aerial Vehicle. International Journal of Communications, 2.

[12] Muharam, A., Mostafa, T. M., \& Hattori, R. (2017, October). Design of power receiving side in wireless charging system for UAV application. In 2017 International Conference on Sustainable Energy Engineering and Application (ICSEEA) (pp. 133-139). IEEE.

[13] Raj, J. S. (2020). A Novel Hybrid Secure Routing for Flying Ad-hoc Networks. Journal of trends in Computer Science and Smart technology (TCSST), 2(03), 155-164.

[14] Sivaganesan, D. Wireless UAV Rotary Wing Communication with Ground Nodes Using Successive Convex Approximation and Energy Saving Mode. Journal on Sustainable Wireless Systems., 02(2), 100107 